\title{
An Effective New Algorithm for Face Recognition
}

\author{
KONG Rui \\ College of Electrical and Information, Jinan University \\ Zhuhai, Guangdong, China,519070 \\ E-mail:tkongrui@jnu.edu.cn
}

\begin{abstract}
To address the influence of the image pre-processing and feature extraction on the recognition rate in face recognition, we propose a algorithm with high recognition rate and fast face recognition under varying illumination environment. The new algorithm deals with complicated illumination conditions of face image, by employing Weber local descriptor (WLD) and Laplacian-of-Gaussian (LoG) descriptor for the extraction of illumination invariant. The processed image is not only insensitive to illumination but also enhances the edge detail information based on complete linear discriminant analysis (CLDA) algorithm for feature extraction. and nearest neighbor based on Euclidean distance classifier. The new algorithm is tested on Yale and the Extended Yale Database B face database respectively. Compared with other face recognition algorithms, the proposed method has outstanding advantage on recognition rate, recognition speed. As a result, It fully meets the real-time requirement for face recognition.
\end{abstract}

Keywords- Weber's Law; Feature extraction; Face recognition.

\section{I . INTRODUCTION}

Currently, automatic face recognition is an important research subject in the field of pattern recognition and artificial intelligence. Although face recognition technology has been in use in many fields, such as video surveillance and entrance control etc, many problems in the research of face recognition still has not been solved. Face recognition systems currently being used usually need individual intervention with facial image acquisition (no expression and frontal face to camera) and fixed environment (fixed illumination), otherwise the recognition system performance will be greatly reduced due to illumination, gesture and expression changing etc. Illumination variation is one of the most significant factors affecting the performance of face recognition and has received much attention in recent years. Many methods have been proposed to handle the of Guangdong Province (No. 2013KJCX0023).

\author{
ZHANG Bing \\ College of Electrical and Information, Jinan University \\ Zhuhai, Guangdong, China,519070 \\ E-mail:tzhangbing@jnu.edu.cn
}

illumination problem. Generally, these methods can be divided into three main categories. The first approach is illumination normalization method, such as Histogram Equalization $(\mathrm{HE})^{[1]}$,logarithm transforms $(\mathrm{LT})^{[2]}$ etc. This method uses image processing technique to preprocess the face image, so the image shows stability under different illumination variation conditions. The second approach is the extraction of illumination invariant features or illumination insensitive measure. The third approach is constructing face model to handle the illumination problem. This method needs light conditions, object shapes and emission characteristic known in advance, and there are too many and strong assumptions, which limits its practical application.

In order to address the influence of illumination variation on recognition rate, this paper proposes a new face recognition algorithm which is composed of three steps. Firstly, we apply weber local description operator ${ }^{[3]}$ operation on face image to get the illumination insensitive feature( WeberFace ${ }^{[4]}, \mathrm{WF}$ ), which can reduce the influence of the illumination to certain extent. Secondly, LoG descriptor is used for edge detection, to extract the edge feature with illumination invariant. Finally, $\operatorname{CLDA}^{[5,6]}$ algorithm is employed for feature extraction and classification. The simulation results show that the proposed method not only significantly improves recognition performance under varying illuminations, but also is faster than other methods.

\section{II . THE NEW FACE RECOGNITION ALGORITHM}

In this paper, we propose a new algorithm to extract the edge feature with illumination invariant to improve the recognition rate. The procedure of new algorithm is as below.

\section{A. Image smoothing based on Gaussion filter}

Laplace operator is sensitive to noise, so firstly we do 
smoothly processing to reduce the influence of noise. We select the Gaussian filter which can obtain good effect here. Assume the original face image is $F(x, y)$, we can obtain new image $F^{\prime}(x, y)$ after applying Gaussian filter. $F^{\prime}(x, y)=F(x, y) * G(x, y, \sigma)$

Where $*$ is the convolution operator and the Gaussian kernel function $G(x, y, \sigma)$ could be expressed as follows:

$$
G(x, y, \sigma)=\frac{1}{2 \pi \sigma^{2}} \exp \left(-\frac{x^{2}+y^{2}}{2 \sigma^{2}}\right)
$$

Where $\sigma$ is the standard deviation. In order to balance the noise reduction and computing time efficiency, based on simulation tests, we choose $\sigma=0.5$ and template size with $3 \times 3$, which can both filter noise and retain the high frequency information of image.

B. The illumination invariant extraction based on Weber's Law and LoG

\section{Weber's Law}

German physiologist Ernst Weber discovered a phenomenon that the ratio of smallest perceptual change in a stimulus and the background level of the stimulus is constant in 1834 , this is the well-known Weber's law.

$$
\frac{\Delta I_{\min }}{I}=k
$$

Where $\Delta I_{\min }$ denotes the adjacent pixel increment relatively, and $I$ denotes the initial strength of the incentive, so $k$ is called Weber fraction and implies that $\Delta I_{\min } / I$ remains constant despite the variations in the $I$ term.

2. Feature extraction based on Weber's law

Chen et al. proposed a local descriptor WLD (Weber Local Descriptor), which consists of two components: differential excitation and orientation to capture the magnitude and the direction of local intensity variations. WLD is also proved to be suitable for the application of texture classification and face detection ${ }^{[3]}$.

The response of the current pixel after transformation can be represented as follows:

$$
\varepsilon\left(X_{c}\right)=\arctan \left(\alpha \sum_{i=0}^{p-1} \frac{X_{c}-X_{i}}{X_{c}}\right)
$$

In order to avoid the large output values, we use the arctangent function, which could partially remove the side-effect of noise. Denote $X_{\mathrm{c}}$ as the center of pixel, and $X_{i}(i=0,1, \ldots, p-1)$ as the neighboring pixels. $p$ is the number of neighbors, and $\alpha$ is a parameter for adjusting the intensity difference between neighboring pixels. Based on experimental analysis, we choose $\alpha=4$, and $\varepsilon\left(X_{c}\right) \in[-\pi / 2, \pi / 2]$.

Based on the Lambertian reflectance model, a face image pixel $F(x, y)$ could be expressed by

$$
F(x, y)=R(x, y) I(x, y)
$$

Where $R(x, y)$ and $I(x, y)$ are the reflectance and the illuminance respectively at each pixel $(x, y), R(x, y)$ depends on the characteristics of the facial surface, which could be regarded as the illumination insensitive part, while $I(x, y)$ depends on the lighting source.

From formula (3)、(4) and (5), we deduce a new face image expression $W F(x, y)$ :

$$
W F(x, y)=\arctan \left[\alpha \sum_{i \in A} \sum_{j \in A} \frac{F(x, y)-F(x-i \Delta x, y-j \Delta y)}{F(x, y)}\right]
$$

In which $A=\{-1,0,1\}$, From (3)、(5), we have

$$
\begin{gathered}
I(x-i \Delta x, y-j \Delta y) \approx I(x, y) \\
F(x-i \Delta x, y-j \Delta y) \approx R(x-i \Delta x, y-j \Delta y) \times I(x-i \Delta x, y-j \Delta y)
\end{gathered}
$$

By substituting (7)、(8) into (6), we could get

$$
W F(x, y)=\arctan \left[\alpha \sum_{i \in A} \sum_{j \in A} \frac{R(x, y)-R(x-i \Delta x, y-j \Delta y)}{R(x, y)}\right]
$$

From the above equation, we could see that $W F(x, y)$ depends only on the reflection component $R(x, y)$, and has nothing to do with the illumination component $I(x, y)$.

Therefore it is an illumination insensitive representation of the original face image $F(x, y)$.

In practical applications, we add a decimal $c=0.001$ in the denominator to avoid $F(x, y)=0$, therefore, the new expression of the illumination insensitive features extracted by WLD is as follows:

$$
W F(x, y)=\arctan \left[\alpha \sum_{i \in A} \sum_{j \in A} \frac{F(x, y)-F(x-i \Delta x, y-j \Delta y)}{F(x, y)+c}\right]
$$

\section{Extracting edge features based on LoG operator}

The idea of Edge detection based on LoG operator originates from biology studies of mammalian visual system. This method processes the different resolution images respectively, on each resolution, and calculates the zero crossing point by the second derivative operator to obtain edge feature. Calculation steps are as follows:

a) Use a two-dimensional Gaussian smoothing template to convolute with target image;

b ) Calculate the Laplacian value of the image after convolution;

c) Detection the zero crossing point in Laplacian image as edge point. 
Based on formula (1) which applies Gaussian smoothing to target image, we have

$$
W F^{\prime}(x, y)=W F(x, y) * G_{o}\left(x, y, \sigma_{o}\right)
$$

Where $W F(x, y)$ is the image after WLD operator process, and the Gaussian kernel function is $G_{o}\left(x, y, \sigma_{o}\right), \sigma_{o}$ is the standard deviation, and $W F^{\prime}(x, y)$ is the image after Gaussian smoothing. Empirically, we choose Gaussian function template $9 \times 9$, and $\sigma_{o}=0.3$ here.

\section{Complete linear discrimination analysis (CLDA)}

Classical Fisher linear discriminant analysis ${ }^{[8]}$ (LDA) is aimed at two kinds of classification problem, with the purpose of selecting the appropriate projection lines to find projection direction which utmost distinguish two types of data points. Although the LDA algorithm achieves good classification effect in image recognition, two problems arise: one is the high dimension vectors operations increase computational complexity, and the other is that the within-class scatter matrix is always singular. In order to address these problems, Fisherface ${ }^{[9]}$ algorithm was proposed which uses principal component analysis (PCA) for dimension reduction before LDA. However, the down side of PCA is the accompanied loss of some important identification information ${ }^{[5]}$. This paper employs fast $\mathrm{PCA}^{[6]}$ method to transform image into PCA space first, then extract the eigenvectors which corresponds to the positive eigenvalues in the scatter matrix. Lastly we apply CLDA algorithm for the second time feature extraction. By doing so, the proposed method can extract more features of identification ability therefore.

\section{EXPERIMENT}

In order to evaluate the effectiveness of the new algorithm, we test it on Yale and the Extended Yale Database $\mathrm{B}^{[10]}$ respectively. The illumination processing methods such as HE $\mathrm{LN}$ and feature extract methods such as classic Fisherface and Fast PCA are compared with the proposed method. Then apply the nearest neighbor classifier.

The Yale database contains 165 images of 15 human subjects under 11 different expressions and illumination conditions. All test images data used in the experiments are manually aligned, cropped to $60 \times 60$ images. For Yale face database, we randomly select five images as training sets, and the rest six images for testing. We carry out five experiments respectively and choose the maximum recognition rate every time, then calculate the average values. The results are shown in TABEL I .

TABEL I the recognition results in Yale database

\begin{tabular}{|c|c|c|c|}
\hline & FastPCA & Fisherface & CLDA \\
\hline ORI & $81.48 \%(55)$ & $84.45 \%(14)$ & $85.19 \%(10)$ \\
\hline HE & $89.63 \%(70)$ & $89.26 \%(13)$ & $91.48 \%(28)$ \\
\hline LN & $95.19 \%(75)$ & $94.07 \%(14)$ & $96.67 \%(15)$ \\
\hline WF & $90.74 \%(75)$ & $88.89 \%(14)$ & $94.81 \%(17)$ \\
\hline WF+LoG & $94.44 \%(65)$ & $92.59 \%(14)$ & $95.92 \%(17)$ \\
\hline
\end{tabular}

We can see from TABEL I (The number in parentheses shows the chosen number of the feature vectors when reaching highest recognition rate) that among many different illumination processing and feature extraction methods ,the $\mathrm{WF}+\mathrm{LoG}+\mathrm{CLDA}$ algorithm proposed in this paper can achieve best recognition effect. The average recognition rate can achieve 96\%. Although LN+CLDA algorithm and the new algorithm is comparable in terms of the recognition rate, when illumination changes drastically, it is inferior to the proposed method in terms of illumination influence. Meanwhile, the recognition rate after CLDA feature extracting exceeds the classical PCA and Fisherface methods. The CLDA algorithm not only can recognize more identification vectors, but also enhance the classification ability of identifying features, and is easier for face recognition.

Next, we perform an experiment in illumination variation serious face database which is named The Extended Yale Database B that contains 380 images of 38 human subjects under 10 different illumination conditions. All test images used in the experiment are manually aligned, cropped to $60 \times 60$ images. We randomly select five images for training, and the rest images for testing. We carry out five experiments respectively, and choose the maximum recognition rate every time, then calculate the average values. The results are shown in TABEL II.

TABEL II the recognition results in the Extended Yale Database B

\begin{tabular}{|c|c|c|c|}
\hline & FastPCA & Fisherface & CLDA \\
\hline ORI & $31.32 \%(90)$ & $66.97 \%(36)$ & $31.19 \%(23)$ \\
\hline HE & $37.24 \%(80)$ & $55.95 \%(37)$ & $45.00 \%(22)$ \\
\hline LN & $87.11 \%(90)$ & $82.24 \%(37)$ & $94.08 \%(72)$ \\
\hline WF & $98.95 \%(40)$ & $97.50 \%(36)$ & $99.60 \%(39)$ \\
\hline WF+LoG & $98.55 \%(85)$ & $93.42 \%(37)$ & $99.74 \%(76)$ \\
\hline
\end{tabular}

We can see from TABEL II (the numbers in parentheses 
show the chosen number of the feature vectors when achieving highest recognition rate) that recognition rate is as low as $31 \%$ by using PCA and CLDA methods, which means illumination variation is one of the important factor to influence face recognition rate. Therefore, it is necessary to reduce the influence of illumination before feature extraction. The LN+CLDA algorithm performs well in the Yale database, but in this experiment its recognition rate is 5 percentage less than the proposed method, and the proposed method reaches the highest recognition rate to $99.74 \%$.As a result, the edge contour of the image can better reflect the raw information after processing through the WF+LoG algorithm. In general the CLDA, WF+LoG+CLDA algorithms have good feature extraction performance on the images under uncontrolled illumination conditions.

Based on the experimental analysis in this paper, the new fusion algorithm for the optimal identification of face image preprocessing and feature extraction has the obvious advantage, mainly manifested in the following aspects: (1) For the face image Gaussian smoothing filter, filtering out the high frequency noise in the image is very useful for retaining the original identification information. It is beneficial for adopting Laplacian-based method to extract insensitive illumination characteristics. (2) For image changes due to complex illumination, we adopt the Weber -based method to extract insensitive illumination characteristics and reduce the impact from complex illumination. Then, using the LoG operator to extract the facial image at the edge, which can be well reflected in the outline of the image, structure and shape characteristics. (3) There exists quite a few shortcomings in the traditional feature extraction methods. For example, the effective features in original images are thrown away in the feature extraction process. As a complement, the proposed method uses complete linear discriminant analysis to extract the features which is obtained through LoG operator operation, and it can extract much more identifying information. We can see that based on the above experimental results, the new algorithm produces outstanding face recognition rate.

\section{CONCLUSION}

In this paper we performed a comprehensive study and discussion aimed at developing a new face recognition algorithm under uncontrolled illumination conditions. Firstly, we select the Gaussian filter to eliminate the high-frequency noise, and obtain WeberFace through Weber local descriptor, which can weaken the shadow or light areas due to the illumination influence. Then, LoG operator is used for extracting the edge feature in face images, which can solve the influence of illumination factor to the classification. Finally, a complete linear discriminant analysis algorithm employs features and nearest neighbor classifier to accomplish the face recognition. The experiments indicate that the proposed method can produce a higher recognition rate than other methods under the uncontrolled illumination conditions at various expression face database. Meanwhile, the new algorithm is fast in terms of computational speed which fully meets the real-time requirement.

\section{REFERENCES}

[1] S. M. Pizer, E. P. Amburn.Adaptive histogram equalization and its variations $[\mathrm{J}]$. Computer Vision Graphics and Image Processing, 1987,39(3):355-368

[2] Savvides M and Kumar V.Illumination normalization using logarithm transforms for face authentication[C] // Proceedings of the 4th International Conference on Audio-and Video-Based Biometric Person Authentication 2003.Berlin:Springer-Verlag,2003:549-556

[3] J. Chen, S. Shan, C.He, G. Zhao, M. Pietikäinen, X. Chen, W. Gao. WLD: A robust local image descriptor [J]. IEEE Transactions on Pattern Analysis and Machine Intelligence,2010,32(9):1705-1720

[4] Biao Wang, Weifeng Li, Wenming Yang, Qingmin Liao. Illumination Normalization Based on Weber's Law With Application to Face Recognition[J]. IEEE Signal Processing Letters,2011,18(8):462-465

[5] Yang J, Yang J Y. Why can LDA be performed in PCA transformed space?[J].Pattern Recognition,2003,36(2):563-566

[6] Yujin Zhang. Subspace-based Face Recognition[M],Beijing:Tsinghua University Press,2009:82-88

[7] $\mathrm{Xu}$ dong Xie, Kin-Man Lam. An efficient illumination normalization method for face recognition[J]. Patterm Recognition Letters, 2006,27:609-617

[8] Fisher R A. The use of multiple measurements in taxonomic problems [J] .Annals of Eugenics ,1936,7(2):179-188

[9] P. N. Belhumeur, J. P. Hespanha, D. J. Kriegman. Eigenfaces vs. Fisherfaces: Recognition using class-specific linear projection[J]. IEEE Transactions on Pattern Analysis and Machine Intelligence, 1997,19(7):711-720

[10]K. C. Lee,J. Ho,D. Kriegman. Acquiring Linear Subspaces for Face Recognition under Variable Lighting[J],IEEE Transactions on Pattern Analysis and Machine Intelligence,2005,27(5):684-698 